\title{
Genotyping of Mycobacterium avium complex organisms using multispacer sequence typing
}

\author{
Correspondence \\ Michel Drancourt \\ Michel.Drancourt@univmed.fr
}

Received 7 August 2009

Revised 14 October 2009

Accepted 19 November 2009

\author{
Caroline Cayrou, ${ }^{1}$ Christine Turenne, ${ }^{2}$ Marcel A. Behr ${ }^{2}$ \\ and Michel Drancourt ${ }^{1} \dagger$
}

${ }^{1}$ Unité de Recherche sur les Maladies Infectieuses et Tropicales Emergentes, UMR CNRS 6236
IRD 3R198, IFR 48 Faculté de Médecine, Université de la Méditerranée, Marseille, France

${ }^{2}$ McGill University Health Centre, Montreal, Quebec, Canada

\section{INTRODUCTION}

Mycobacterium avium complex (MAC) comprises a growing number of species of slowly growing environmental and animal-associated mycobacteria responsible for opportunistic human infections (Turenne et al., 2006). Mycobacterium avium comprises M. avium subsp. hominissuis, an environmental species responsible for opportunistic infections in pigs and humans including HIVinfected, immunocompromised patients (Komijn et al.,

tPresent address: Unité des Rickettsies, Faculté de Médecine 27, Boulevard Jean Moulin 13385, Marseille cedex 5, France.

Abbreviations: ITS-1, intergenic transcribed spacer 16S-23S rDNA region; MAC, Mycobacterium avium complex; MST, multispacer sequence typing; ST, spacer type.

The GenBank/EMBL/DDBJ accession numbers for the genotype sequences of the isolates under study are FJ417966-FJ417968, FJ417971-FJ417998, FJ418000-FJ418007, FJ418009-FJ418034, FJ418037-FJ418044 and FJ892702-FJ892715.

A supplementary table and two supplementary figures are available with the online version of this paper.
1999; Mijs et al., 2002), and clones evolved from this subspecies, namely $M$. avium subsp. avium, M. avium subsp. silvaticum and M. avium subsp. paratuberculosis (Harris \& Barletta, 2001; Thorel et al., 1990; Turenne et al., 2008). Mycobacterium intracellulare is an environmental organism responsible for enlarged lymph nodes in children and progressive pulmonary disease in elderly women (Kyriakopoulos et al., 1997). The analysis of the intergenic transcribed spacer 16S-23S rDNA region (ITS-1) has aided in the recognition of the species Mycobacterium chimaera, incorporating sequevar MAC-A organisms isolated from patients with pulmonary cavitations, pulmonary abscess, chronic obstructive pulmonary disease and bronchiectasis (Tortoli et al., 2004; Bills et al., 2009), and of the species Mycobacterium colombiense, incorporating sequevar MAC$\mathrm{X}$ organisms isolated from the blood and sputum of HIVinfected patients in Colombia (Murcia et al., 2006) and from diseased lymph nodes in children (Esparcia et al., 2008; Vuorenmaa et al., 2009). Mycobacterium arosiense was recently described in an immunocompromised child with disseminated osteomyelitic lesions (Bang et al., 2008). 
The analysis of the partial $r p o B$ gene sequence further distinguished Mycobacterium bouchedurhonense, Mycobacterium marseillense and Mycobacterium timonense organisms isolated from patients with pulmonary disease (Ben Salah et al., 2009).

MAC organisms have been widely isolated from inanimate environments such as soil and water, including drinking water (Covert et al., 1999; Falkinham et al., 2001; Le Dantec et al., 2002; Santos et al., 2005; Hilborn et al., 2008) and water from a hospital water distribution system (Du Moulin et al., 1988). Several animals, including birds, pigs, cattle and sheep, could be also colonized or infected by MAC organisms (Biet et al., 2005). Despite the fact that several investigations found a statistically significant correlation between the exposure of patients to fresh water and MAC infection, demonstration of a microbiological link was lacking (Nishiuchi et al., 2007; Reed et al., 2006) or yielded only partial evidence of direct transmission (Hilborn et al., 2008). Therefore, the source of MAC organisms responsible for infections in most patients remains poorly understood.

One limitation in understanding the source of exposure and risk of disease due to MAC species has been in part a lack of a genotyping method applicable to all organisms belonging to the MAC. Sequencing the 16S rRNA gene is only of value for the identification of MAC at the species level. Partial $r p o B$ gene sequencing identified all species and $M$. avium subspecies but was not able to genotype MAC isolates (Ben Salah et al., 2008). In fact, all methods have been applied to only a few MAC species (Blackwood et al., 2000; Cilliers et al., 1997; Kasai et al., 2000; Morita et al., 2004; Thierry et al., 1993; Tortoli et al., 2004; Turenne et al., 2006, 2007, 2008) including time-consuming RFLP analysis (Guerrero et al., 1995) and PFGE (Mazurek et al., 1993; Bruijnesteijn van Coppenraet et al., 2008; PestelCaron et al., 1999).

In order to gain further knowledge of MAC species populations, in this study we developed multispacer sequence typing (MST), a new method for the genotyping of MAC organisms, based on the sequence analysis of four variable intergenic spacers. MST combines the advantages of PCR and sequencing, and allows the limitations of previous genotyping methods to be circumvented.

\section{METHODS}

Mycobacterium strains. Reference strains (see Supplementary Table S1, available with the online version of this paper) M. avium subsp. avium ATCC $25291^{\mathrm{T}}$, M. avium subsp. silvaticum ATCC $49884^{\mathrm{T}}$, M. avium subsp. paratuberculosis ATCC $19698^{\mathrm{T}}$ and $M$. intracellulare ATCC 15985 were purchased from the American Tissue Culture Collection (ATCC; Manassas, VA, USA). M. chimaera DSM $44623^{\mathrm{T}}$ and $M$. arosiense DSM $45069^{\mathrm{T}}$ were purchased from the German Collection of Micro-organisms (DSMZ, Braunschweig, Germany). M. avium subsp. hominissuis IWGMT 49, a pig isolate, was kindly provided by Dick van Soolingen (National Institute of Public Health and the Environment, Bilthoven, The Netherlands).
M. colombiense CIP $108962^{\mathrm{T}}$ was purchased from the Institut Pasteur Collection, Paris, France. M. bouchedurhonense CSUR P34 ${ }^{\mathrm{T}}$ (Collection de Souches de l'Unité des Rickettsies), M. marseillense CSUR P $30^{\mathrm{T}}$ and $M$. timonense CSUR $\mathrm{P} 32^{\mathrm{T}}$ were previously isolated and characterized in our laboratory (Ben Salah et al., 2009). We further studied $64 \mathrm{MAC}$ clinical isolates preserved on beads at $-20{ }^{\circ} \mathrm{C}$ in our clinical microbiology laboratory in 1997-2008 (Supplementary Table S1). These isolates were previously identified by a polyphasic approach based on PCR amplification and sequencing of $r p o B, 16 \mathrm{~S}$ rRNA and $h s p 65$ genes and ITS-1. For this study, each isolate was inoculated into Middlebrook 7H9 liquid medium in the BACTEC 9000MB apparatus (Becton Dickinson) and subcultured onto Middlebrook $7 \mathrm{H} 10$ agar (Becton Dickinson) at $30{ }^{\circ} \mathrm{C}$. Purity was confirmed by microscopic examination after Ziehl-Neelsen staining. DNA was extracted from colonies using the QIAamp mini kit (Qiagen) according to the manufacturer's instructions, after inactivation by Chelex and heating at $96{ }^{\circ} \mathrm{C}$ for $1 \mathrm{~h}$. We further analysed the DNA extracted from 31 MAC organisms in the M. A. Behr laboratory, McGill University, Canada, including 15 MAC isolates provided by Des Collins (AgResearch, NCBID Wallaceville, Upper Hutt, New Zealand) (Supplementary Table S1). These isolates were previously characterized by hsp65 gene sequencing, insertionsequence typing, RFLP and large-sequence polymorphism (LSP) regions analysis (Turenne et al., 2008).

Selection of intergenic spacers and primer design. The sequences of intergenic spacers were obtained after alignment of the M. avium subsp. hominissuis strain 104 (GenBank accession number NC_008595) and M. avium subsp. paratuberculosis strain K-10 (GenBank accession number NC_002944) genomes. Intergenic spacer sequences were then aligned by using the ssaha2 software (Ning et al., 2001) and were ranked by size from $100 \mathrm{bp}$ to $1000 \mathrm{bp}$ and by percentage of sequence similarity. The selection of spacers was done according to the following criteria: (i) a spacer sequence size comprised between $300 \mathrm{bp}$ and $800 \mathrm{bp}$, in line with the capabilities of a single capillary sequencer run, and (ii) a number of variable bases $<50$ within each of the 16 spacers in order to increase the probability that primers designed for the $M$. avium subspecies would also target other MAC species. PCR primers were designed using Primer3 software (http://fokker.wi.mit.edu). The specificity of PCR primers was predicted by comparing their sequences with those deposited in GenBank using BLASTN software (http://www.ncbi.nlm.nih.gov).

PCR amplification and sequencing of spacers. PCRs were carried out in a Biometra thermocycler (LABGENE Scientific Instruments). The PCR mixture $(50 \mu \mathrm{l})$ contained $5 \mu \mathrm{l} 10 \times$ Taq buffer, $200 \mu \mathrm{M}$ of each dNTP, $2.5 \mu l 25 \mathrm{mM} \mathrm{MgCl}_{2}, 1.25 \mathrm{U}$ HotStarTaq polymerase (Qiagen), $1 \mu \mathrm{l} 10 \mathrm{pM}$ each primer (Eurogentec) and $5 \mu$ l purified DNA. Amplification was carried out using the following conditions: an initial 15 min denaturation at $95{ }^{\circ} \mathrm{C}$ was followed by 40 cycles of denaturation for $30 \mathrm{~s}$ at $95{ }^{\circ} \mathrm{C}$, annealing for $30 \mathrm{~s}$ at $60{ }^{\circ} \mathrm{C}$ and extension for $1 \mathrm{~min}$ at $72{ }^{\circ} \mathrm{C}$; amplification was completed by holding the reaction mixture for $5 \mathrm{~min}$ at $72{ }^{\circ} \mathrm{C}$. Sterile water was used as a negative control in each PCR assay. PCR products were visualized under UV illumination after electrophoresis on a $1.5 \%$ agarose gel stained with ethidium bromide. PCR products were purified by using a MultiScreen PCR filter plate as recommended by the manufacturer (Millipore). Forward and reverse sequencing mixtures contained $3 \mu \mathrm{l}$ buffer (BigDye V1, Applied Biosystems), $2 \mu 1$ BigDye Terminator reaction mix, $5 \mu$ distilled $\mathrm{H}_{2} \mathrm{O}$ and $1 \mu \mathrm{l}$ of 10 pmol primer $\mu^{-1}$, in a final volume of $15 \mu$ l. The sequencing reaction comprised an initial $1 \mathrm{~min}$ denaturation step at $95{ }^{\circ} \mathrm{C}$ followed by 25 cycles of denaturation at $96{ }^{\circ} \mathrm{C}$ for $10 \mathrm{~s}$, annealing at $50{ }^{\circ} \mathrm{C}$ for $5 \mathrm{~s}$ and elongation at $60{ }^{\circ} \mathrm{C}$ for $3 \mathrm{~min}$. Sequencing products were purified using Sephadex plates (Amersham Biosciences), centrifuged at $910 \mathrm{~g}$ for $6 \mathrm{~min}$, and purified sequencing products were deposited on a MicroAmp Optical 96-well reaction plate (Applied Biosystems). 
Sequencing electrophoresis was performed on a 3130 Genetic Analyzer (Applied Biosystems).

Multiple spacer sequence analysis. Nucleotide sequences were edited using the AutoAssembler 2.1 software (Applied Biosystems). We determined the Hunter-Gaston index (Hunter \& Gaston, 1988) for $M$. avium, $M$. intracellulare, $M$. chimaera and $M$. colombiense isolates (this was not done for the other species comprising only one isolate). Multiple alignments of sequences were carried out using the CLUSTAL_X software (Thompson et al., 1997). Phylogeny reconstruction of all sequence alignments was performed in MEGA 3.1 using the neighbour-joining and bootstrapping methods (Kumar et al., 2004). Each sequence was translated in silico with the web software ExPASy translate tool (http://www.expasy.ch) in order to verify the presence of potential transcripts. The Mycobacterium tuberculosis $\mathrm{H} 37 \mathrm{Rv}$ genome sequence (GenBank accession number NC 000962) was used as an out-group root.

Stability assay. In order to check the stability of intergenic spacer sequences, we repeated MST genotyping as described above after two subcultures for isolate numbers 7, 10, 13/00, 59 and 71, $M$. intracellulare ATCC 15985, M. avium subsp. paratuberculosis strain K-10 and M. avium subsp. paratuberculosis ATCC $19698^{\mathrm{T}}$, randomly chosen for this purpose.

Reference IS1245-RFLP typing. Two M. avium subsp. hominissuis isolates were randomly selected in each one of the MST subclusters determined in this study in order to analyse them by reference IS1245-RFLP typing performed as previously described (Turenne et al., 2008) and to compare IS1245-RFLP typing with MST genotyping.

Discrimination power. The discrimination power of the MST and IS 1245-RFLP genotyping methods was calculated using the HunterGaston index, which was estimated as

$D=1-\frac{1}{N(N-1)} \sum_{j=1}^{S} n_{j}\left(n_{j}-1\right)$

where $N$ was the total number of isolates in the sample population, $S$ was the total number of types described, and $n_{j}$ is the number of isolates belonging to the $j$ th type (Hunter \& Gaston, 1988).
Nucleotide sequence accession numbers. Each genotype sequence was deposited in the GenBank database under accession numbers FJ417966-FJ417968, FJ417971-FJ417998, FJ418000-FJ418007, FJ418009-FJ418034, FJ418037-FJ418044 and FJ892702-FJ892715 and in our local database (http://ifr48.timone.univ-mrs.fr/portail2/).

\section{RESULTS}

\section{Spacer sequencing}

Of 621 intergenic spacers $(<800 \mathrm{bp})$ detected via alignment of the genomic sequence of $M$. avium subsp. hominissuis strain 104 with that of $M$. avium subsp. paratuberculosis strain K-10, 16 spacers fulfilled our selection criteria. PCR primers could be designed for only 11 spacers as the sequence of five spacers was not conserved enough to allow for PCR primer design. After tentative PCR amplification of the spacers on seven reference strains including the $M$. avium subspecies, $M$. intracellulare, $M$. chimaera and M. colombiense, seven spacers could not be amplified in all reference strains. Further analyses were therefore undertaken using the four spacers herein referred to as MST2, MST4, MST15 and MST16 (Table 1). Spacer MST16, a 595-674 bp spacer, was found to be the most variable spacer, classifying the 106 isolates under study into 24 genotypes. PCR amplification of MST4 yielded a product size between $360 \mathrm{bp}$ for $M$. colombiense CIP $108962^{\mathrm{T}}$ and $473 \mathrm{bp}$ for clinical isolate no. 75 and classified the 106 isolates into 23 genotypes. Spacer MST2 classified the 106 isolates into 23 genotypes, with a product size between $611 \mathrm{bp}$ for M. avium subsp. hominissuis IWGMT 49 and $646 \mathrm{bp}$ for clinical isolate no. 13/00. PCR amplification of MST15 yielded a product size of between $338 \mathrm{bp}$ for isolate no. 43 and $405 \mathrm{bp}$ for isolate no. 47 and classified the $106 \mathrm{MAC}$ isolates into 17 genotypes. For any spacer, the differences in sequence between isolates were due to mutations, deletions and insertions.

Table 1. Spacer characteristics and the primers used in this study

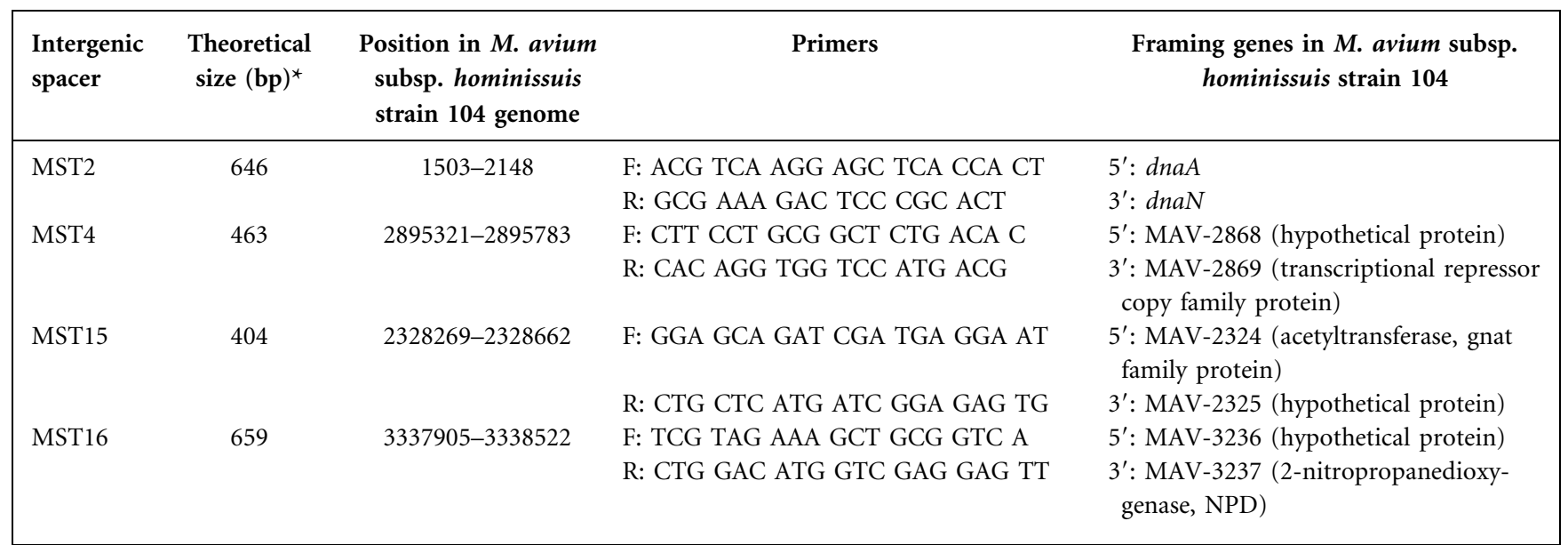

*Theoretical size of PCR products was determined according to the position primers within M. avium subsp. hominissuis strain 104 (GenBank accession number NC_008595) genome. 


\section{MST genotyping}

Combining the four spacer sequences obtained in 106 MAC isolates, including the reference strains, yielded 45 spacer types (STs), with an index of discrimination of 0.94 (Table 2). Thirty-five of the 45 types (STs $1-5,9,12$, $14-19,21-23,25-32,36,38-45)$ contained one isolate each; three STs (nos 24, 34 and 37) contained two isolates each; ST-8 contained three isolates; STs 7, 33 and 35 contained four isolates; ST-20 contained five isolates; STs 11 and 13 contained seven isolates each; ST-6 contained 12 isolates; and ST-10 contained 21 isolates. Each ST was species-specific: ST-31 and ST-32 were specific for $M$. colombiense; ST-33 and ST-34 were specific for M. chimaera; ST-35 to ST-41 were specific for M. intracellulare; and ST-1 to ST-30 were specific for $M$. avium. In the latter species, each ST was subspecies specific: ST-1 was specific for M. avium subsp. silvaticum; ST-2 to ST-5 were specific for M. avium subsp. avium; ST-6 to ST-9 were specific for M. avium subsp. paratuberculosis; ST-10 to ST-30 were specific for $M$. avium subsp. hominissuis. ST-42 to ST-45 were specific for $M$. bouchedurhonense, $M$. marseillense, $M$. timonense and $M$. arosiense, respectively. The Hunter-Gaston index was 1 in M. colombiense (2 strains), 0.90 in M. avium ( 83 strains), 0.87 in $M$. intracellulare (11 strains) and 0.53 in M. chimaera (6 strains). As for the M. avium subspecies, the Hunter-Gaston index was 1 in $M$. avium subsp. avium (4 strains), 0.84 in $M$. avium subsp. hominissuis (58 strains) and 0.60 in M. avium subsp. paratuberculosis (20 strains).

\section{Stability assay}

After two subcultures, spacer sequences determined were: ST-17 for isolate no. 10; ST-18 for isolate no. 13/00; ST-14 for isolate no. 59; ST-21 for isolate no. 71; ST-16 for isolate no. 7; ST-35 for M. intracellulare; and ST-6 for the two strains of $M$. avium subsp. paratuberculosis. The spacer sequences determined for these eight isolates after two subcultures were identical to those determined before subculturing.

\section{Phylogenetic tree}

The phylogenetic tree based on the concatenation of the four intergenic spacer sequences separated the MAC species into two principal clusters. One cluster comprised all the $M$. avium isolates and a second cluster comprised M. intracellulare, M. chimaera, M. timonense, M. bouchedurhonense, M. marseillense, M. arosiense and M. colombiense isolates (Fig. 1). The latter cluster was divided into one subcluster comprising $M$. colombiense and $M$. timonense isolates with a $100 \%$ bootstrap value and other subclusters comprising $M$. chimaera, $M$. intracellulare, $M$. bouchedurhonense and $M$. marseillense isolates with a $100 \%$ bootstrap value. The M. avium cluster comprised six subclusters.

\section{Comparison between reference RFLP and MST genotyping}

We compared MST and IS1245 genotyping for 15 strains: three M. avium subsp. avium (ATCC 25291, ATCC 35713 and ATCC 15769) and $12 \mathrm{M}$. avium subsp. hominissuis (ATCC 700898, DV, 104, 76102, 28132, MB 63764, MB $64490,26 / 05,10,33,59$ and 71 ). As for M. avium subsp. hominissuis, we observed that strains with different STs had different RFLP profiles (Supplementary Figs S1 and S2). Therefore, strains with STs 11, 10, 29, 20, 25, 17, 18, 14 and 21 had distinct RFLP profiles. Strains DV and MB 63764 had the same ST-10 but exhibited different RFLP profiles (>3 bands difference). Likewise, strains 700898 and 104 (ST no. 11; 4 bands difference), and 28132 and MB64490 (ST no. 20; 5 bands difference), had similar but distinct RFLP profiles. For STs 29, 25, 17, 18, 14 and 21 only one strain was tested.

As has previously been described, IS 1245 genotyping on $M$. avium subsp. avium (Turenne et al., 2007) showed the expected three-band pattern with the same position for each band.

\section{DISCUSSION}

Selection bias of MAC isolates was minimized as we included clinical isolates from eight distinct countries, i.e. France, Canada, New Zealand, Australia, UK, Faroe Islands, USA and Colombia. However, we analysed only clinical isolates from human or animal sources and the application of MST to isolates collected from the inanimate environment remains to be studied. We observed that spacer sequence variability was due to mutations, deletions and insertions. We did not observe repeat variation or variable number tandem repeats, contrary to what is observed in Mycobacterium tuberculosis complex species (Djelouadji et al., 2008a, b) and Rickettsia species (Fournier \& Raoult, 2007).

The identification of recently described MAC species relied on 16S rRNA gene sequencing (Tortoli et al., 2004; Murcia et al., 2006; Ben Salah et al., 2009; Bang et al., 2008). However, only one base (position 403) discriminates between $M$. intracellulare and M. chimaera, with 97.8$99.7 \%$ 16S rRNA gene sequence identity among the different MAC species. We observed that each MAC isolate contained one or more STs whereas each ST was found in only one species. Despite the fact that MST discriminated most of the $M$. chimaera isolates from the $M$. intracellulare isolates, nevertheless MST provided limited discrimination among the $M$. chimaera isolates. In addition, $h s p 65, r p o B$ and ITS- 1 sequencing were further used as a basis for the description of $M$. chimaera and $M$. colombiense and to complete that of $M$. arosiense, $M$. bouchedurhonense, $M$. marseillense and M. timonense (Murcia et al., 2006; Tortoli et al., 2004; Bang et al., 2008; Ben Salah et al., 2009). In particular, the description of M. chimaera mainly relied on ITS-1 sequencing. Unsurprisingly, the percentage of ITS-1 
Table 2. List of MST types identified

Each MST allele was determined by sequencing the corresponding spacer. The combination of spacer genotypes, strains and species included are presented for each MST type. M.a.a., M. avium subsp. avium; M.a.s., M. avium subsp. silvaticum; M.a.h., M. avium subsp. hominissuis; M.a.p., M. avium subsp. paratuberculosis; C, cattle genotype; S, sheep genotype; M.i., M. intracellulare; M.ch., M. chimaera; M.co., M. colombiense; M.m., M. marseillense; M.t., M. timonense; M.b., M. bouchedurhonense; M.a., M. arosiense.

\begin{tabular}{|c|c|c|c|c|c|c|c|}
\hline \multirow[t]{2}{*}{ ST } & \multicolumn{4}{|c|}{ Genotypes } & \multirow[t]{2}{*}{ Strains } & \multirow[t]{2}{*}{ Species } & \multirow{2}{*}{$\begin{array}{l}\text { Total no. of } \\
\text { strains }\end{array}$} \\
\hline & MST2 & MST4 & MST15 & MST16 & & & \\
\hline 2 & 1 & 2 & 1 & 2 & ATCC $25291^{\mathrm{T}}$ & M.a.a. & 1 \\
\hline 3 & 2 & 3 & 1 & 2 & ATCC 35718 & M.a.a. & 1 \\
\hline 4 & 1 & 1 & 1 & 2 & ATCC 15769 & M.a.a & 1 \\
\hline 6 & 4 & 4 & 2 & 3 & $\begin{array}{l}\text { ATCC } 19698^{\mathrm{T}} ; \mathrm{K}-10 ; 04-4531 ; 119 ; \text { ATCC } 43015 ; \text { TMC 1613; } \\
\text { 5979; 7428; 87/8880; 6601; 316F; } 7296\end{array}$ & M.a.p. C & 12 \\
\hline 7 & 5 & 4 & 2 & 3 & $6758 ; 6756 ; \mathrm{P} 133 / 79 ; 6759$ & M.a.p. S & 4 \\
\hline 8 & 6 & 4 & 2 & 4 & $86-45 ;$ LN20; 85/14 & M.a.p. S & 3 \\
\hline 9 & 6 & 5 & 2 & 4 & P465 & M.a.p. S & 1 \\
\hline 10 & 1 & 8 & 1 & 7 & $\begin{array}{l}\text { IWGMT } 49 ; 45 ; 46 ; 14 / 00 ; 251544 ; 251545 ; 251548 ; 52 / 07 ; \text { DV; } \\
\text { MB } 63764 ; 5 ; 9 ; 12 ; 13 ; 23 ; 26 ; 52 ; 63 ; 81 ; 86 / 03 ; 49\end{array}$ & M.a.h. & 21 \\
\hline 13 & 1 & 9 & 1 & 8 & $43 / 00 ; 48 / 04 ; 55 / 06 ; 31 ; 60 ; 75 ; 6$ & M.a.h. & 7 \\
\hline 14 & 1 & 10 & 1 & 9 & 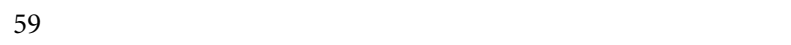 & M.a.h. & 1 \\
\hline 15 & 1 & 11 & 6 & 10 & 43 & M.a.h. & 1 \\
\hline 16 & 9 & 11 & 6 & 10 & 7 & M.a.h. & 1 \\
\hline 17 & 1 & 12 & 7 & 8 & 10 & M.a.h. & 1 \\
\hline 18 & 10 & 13 & 4 & 6 & $13 / 00$ & M.a.h. & 2 \\
\hline 19 & 11 & 12 & 4 & 11 & 42 & M.a.h. & 1 \\
\hline 20 & 1 & 13 & 1 & 7 & $39 ; 50 ; 61 ; 28132 ;$ MB64490 & M.a.h. & 5 \\
\hline 21 & 1 & 14 & 8 & 6 & 71 & M.a.h. & 1 \\
\hline 22 & 1 & 9 & 1 & 12 & $5 / 06$ & M.a.h. & 1 \\
\hline 23 & 1 & 8 & 1 & 13 & $6 / 05$ & M.a.h. & 1 \\
\hline 24 & 1 & 9 & 1 & 7 & $7 / 01$ & M.a.h. & 1 \\
\hline 32 & 13 & 17 & 11 & 15 & $98 / 02$ & M.co. & 1 \\
\hline 33 & 14 & 18 & 12 & 16 & DSM $44623^{\mathrm{T}} ; 29 / 06 ; 3 / 08 ; 73 / 05$ & M.ch. & 4 \\
\hline 34 & 15 & 19 & 12 & 17 & $251556 ; 251550$ & M.ch. & 2 \\
\hline 35 & 16 & 20 & 13 & 18 & ATCC $15985 ; 251542 ; 17 / 03 ;$ ATCC $13950^{\mathrm{T}}$ & M.i. & 4 \\
\hline 36 & 17 & 20 & 13 & 19 & 251540 & M.i. & 1 \\
\hline 37 & 8 & 20 & 13 & 20 & $251541 ; 55 / 03$ & M.i. & 2 \\
\hline 38 & 18 & 21 & 13 & 17 & 251554 & M.i. & 1 \\
\hline 39 & 14 & 20 & 14 & 16 & 79 & M.i. & 1 \\
\hline 40 & 19 & 22 & 15 & 21 & $27 / 04$ & M.i. & 1 \\
\hline 41 & 20 & 20 & 16 & 17 & ATCC 35761 & M.i. & 1 \\
\hline 42 & 21 & 23 & 17 & 22 & CIP $109828^{\mathrm{T}}$ & M.m. & 1 \\
\hline 43 & 22 & 24 & 18 & 23 & CIP $109830^{\mathrm{T}}$ & M.t. & 1 \\
\hline 44 & 23 & 25 & 19 & 24 & CIP $109827^{\mathrm{T}}$ & M.b. & 1 \\
\hline 45 & 24 & 0 & 0 & 25 & DSM $45069^{\mathrm{T}}$ & M.a. & 1 \\
\hline
\end{tabular}




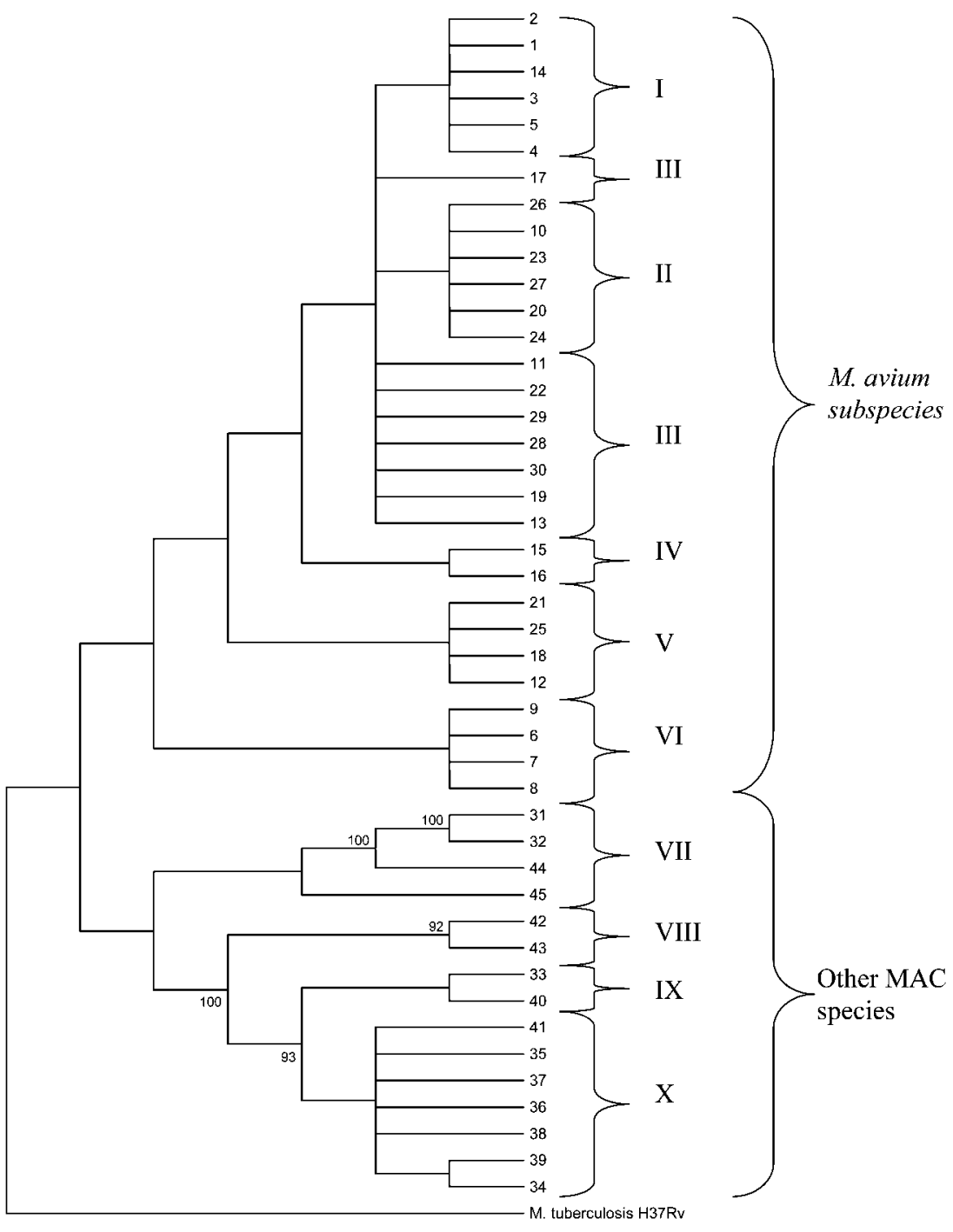

Fig. 1. Tree based on concatenated sequences of four intergenic spacers for each MST type. The topology was calculated using bootstrap values derived from 1000 repetitions (see Methods). Only bootstrap values $>90 \%$ are indicated. Mycobacterium tuberculosis H37Rv sequences were used as root. The Arabic numbers refer to the ST number as indicated in Table 2. Roman numbers refer to clusters formed by the bracketed STs. 'Other MAC species' are $M$. intracellulare, $M$. chimaera, $M$. colombiense, $M$. bouchedurhonense, $M$. timonense, $M$. arosiense and $M$. marseillense.

sequence similarity values was in the same range as that we observed for MST, both systems being based on spacer sequencing. Because of the possible difficulty in resolving $M$. intracellulare and $M$. chimaera isolates and the fact that ITS-1, hsp65 and rpoB gene sequencing can identify all MAC species, MST may not be the most efficient tool for the accurate identification of any MAC isolate.

We further observed that some STs were found in only one of the four M. avium subspecies, suggesting that MST could be used for the identification of $M$. avium subspecies. In particular, MST analysis found one ST specific for M. avium subsp. silvaticum; however, whether a single spacer sequence, including a unique ST, is sufficient to support naming an organism as a unique subspecies requires further study (Turenne et al., 2008). Indeed, multi-locus sequence analysis (MLSA) found that the number of single nucleotide polymorphisms (SNP) separating M. avium subsp. silvaticum from $M$. avium subsp. avium $(n=4)$ was similar to the number of SNPs found within $M$. avium subsp. avium $(n=6)$. In our study, we analysed only one M. avium subsp. silvaticum by MST; nevertheless, this isolate exhibited a unique MST profile, as was also found using MLSA (Turenne et al., 2008). MST analysis, being based on spacer sequencing, may indeed reveal a higher diversity than MLSA, which is based on gene sequencing, because less evolutionary pressure on spacers could yield higher sequence diversity. This point warrants further analysis. We further observed a high degree of diversity in M. avium subsp. hominissuis clinical isolates; sequence identity varied from $96.3 \%$ to $99.9 \%$. Some spacers exhibited a higher degree of sequence diversity among the M. avium subsp. hominissuis isolates than among isolates belonging to other $M$. avium subspecies. This observation agrees with previous observations of heterogeneity among M. avium subsp. hominissuis isolates (Turenne et al., 2008), and suggests that this group of organisms warrants further taxonomic investigation.

In this study, 106 MAC isolates belonging to eight MAC species were separated into 45 STs with an index of discrimination of 0.94. Moreover, we observed that sequences were stable after two subcultures; indeed, we 

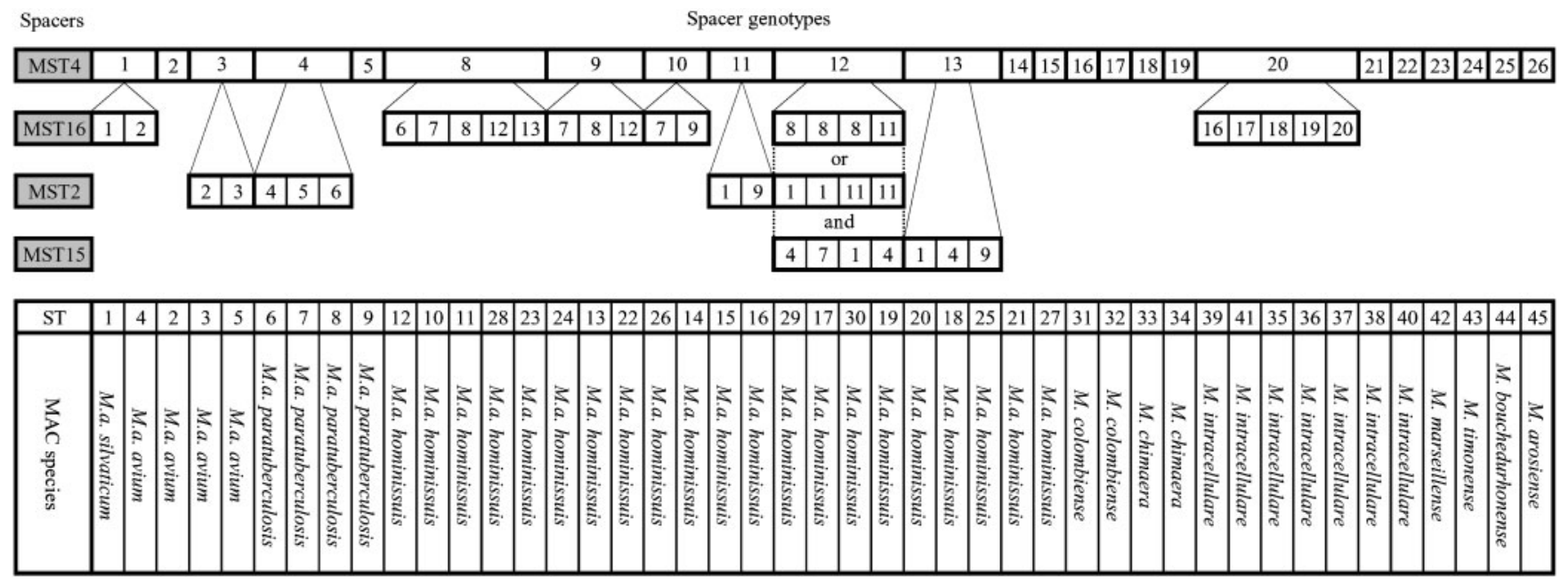

Fig. 2. Guidelines for selection of spacers for MST genotyping of MAC isolates.

obtained the same genotype for the isolates tested. This is of interest, as no method has been published for the genotyping of all organisms belonging to any one of the eight MAC species. Indeed, sequencing of the $h s p 65$ gene or ITS- 1 , and the MLSA method were restricted to only some of the MAC species (Murcia et al., 2006; Turenne et al., 2006, 2008). No genotyping method has been published for the recently described $M$. colombiense, $M$. chimaera, $M$. timonense, M. bouchedurhonense, M. marseillense and $M$. arosiense species. MST is therefore the first method published for the genotyping of any MAC isolate, regardless of the species it belongs to. The analysis of RFLP associated with IS1245 and PFGE, two reference methods for MAC genotyping, are time-consuming methods that require a large amount of mycobacterial DNA and provide comparative, rather than stand-alone results. MST, being based on PCR amplification as a first step, circumvents this limitation and allows genotyping starting from a single colony. Consistent with the different applications of classification methods (such as MST) and strain-tracking methods (such as RFLP), we observed that different MST types had different RFLP profiles but that inside a MST type the strains could have different RFLP profiles. These findings indicate that these methods are complementary. For identifying the species/subspecies of an organism, MST is simple and practical. When comparing two isolates, for instance looking for transmission or testing for clinical relapse, a method such as RFLP is likely to be more discriminatory. Although sequencing all spacers was necessary to identify all MST types, STs 17 , 19,29 and 30 could be defined by three spacers only, for STs $1,3-8,10-16,18,20,22-26,28,35-37,39$ and 41 only two spacers were necessary, and for STs 2, 9, 21, 27, 31-34, 38 and $40-45$ only one spacer was sufficient. Therefore, the MST genotyping could be simplified by first determining the sequence of spacer MST4, followed by the analysis of other spacers depending on the initial result (Fig. 2).
In conclusion, MST was unique in being able to classify MAC isolates into species, M. avium subspecies and strains. We propose MST as a suitable method to genotype any MAC organism after species and subspecies identification.

\section{REFERENCES}

Bang, D., Herlin, T., Stegger, M., Andersen, A. B., Torkko, P., Tortoli, E. \& Thomsen, V. O. (2008). Mycobacterium arosiense sp. nov., a slowly growing, scotochromogenic species causing osteomyelitis in an immunocomprised child. Int J Syst Evol Microbiol 58, 2398-2402.

Ben Salah, I., Adekambi, T., Raoult, D. \& Drancourt, M. (2008). rpoB sequence-based identification of Mycobacterium avium complex species. Microbiology 154, 3715-3723.

Ben Salah, I., Cayrou, C., Raoult, D. \& Drancourt, M. (2009). Mycobacterium marseillense sp. nov., Mycobacterium timonense sp. nov. and Mycobacterium bouchedurhonense sp. nov., novel species in the Mycobacterium avium complex. Int J Syst Evol Microbiol 59, 28032808.

Biet, F., Boschiroli, M. L., Thorel, M. F. \& Guilloteau, L. A. (2005). Zoonotic aspects of Mycobacterium bovis and Mycobacterium aviumintracellulare complex (MAC). Vet Res 36, 411-436.

Bills, N. D., Hinrichs, S. H., Aden, T. A., Wickert, R. S. \& Iwen, P. C. (2009). Molecular identification of Mycobacterium chimaera as a cause of infection in a patient with chronic obstructive pulmonary disease. Diagn Microbiol Infect Dis 63, 292-295.

Blackwood, K. S., He, C., Gunton, J., Turenne, C. Y., Wolfe, J. \& Kabani, A. M. (2000). Evaluation of recA sequences for identification of Mycobacterium species. J Clin Microbiol 38, 2846-2852.

Bruijnesteijn van Coppenraet, L. E., de Haas, P. E., Lindeboom, J. A., Kuijper, E. J. \& van Soolingen, D. (2008). Lymphadenitis in children is caused by Mycobacterium avium hominissuis and not related to 'bird tuberculosis'. Eur J Clin Microbiol Infect Dis 27, 293-299.

Cilliers, F. J., Warren, R. M., Hauman, J. H., Wiid, I. J. \& van Helden, P. D. (1997). Oligonucleotide (GTG) 5 as an epidemiological tool in the study of nontuberculous mycobacteria. J Clin Microbiol 35, 1545-1549.

Covert, T. C., Rodgers, M. R., Reyes, A. L. \& Stelma, G. N., Jr (1999). Occurrence of nontuberculous mycobacteria in environmental samples. Appl Environ Microbiol 65, 2492-2496. 
Djelouadji, Z., Arnold, C., Gharbia, S., Raoult, D. \& Drancourt, M. (2008a). Multispacer sequence typing for Mycobacterium tuberculosis genotyping. PLoS One 3, e2433.

Djelouadji, Z., Raoult, D., Daffé, M. \& Drancourt, M. (2008b). A single step sequencing method for identification of Mycobacterium tuberculosis complex species. PLoS Negl Trop Dis 2, e253.

Du Moulin, G. C., Stottmeier, K. D., Pelletier, P. A., Tsang, A. Y. \& Hedley-Whyte, J. (1988). Concentration of Mycobacterium avium by hospital hot water systems. JAMA 260, 1599-1601.

Esparcia, O., Navarro, F., Quer, M. \& Coll, P. (2008). Lymphadenopathy caused by Mycobacterium colombiense. J Clin Microbiol 46, 1885-1887.

Falkinham, J. O., III, Norton, C. D. \& LeChevallier, M. W. (2001). Factors influencing numbers of Mycobacterium avium, Mycobacterium intracellulare, and other mycobacteria in drinking water distribution systems. Appl Environ Microbiol 67, 1225-1231.

Fournier, P. E. \& Raoult, D. (2007). Identification of rickettsial isolates at the species level using multi-spacer typing. BMC Microbiol 7, 72.

Guerrero, C., Bernasconi, C., Burki, D., Bodmer, T. \& Telenti, A. (1995). A novel insertion element from Mycobacterium avium, IS 1245, is a specific target for analysis of strain relatedness. J Clin Microbiol 33, 304-307.

Harris, N. B. \& Barletta, R. G. (2001). Mycobacterium avium subsp. paratuberculosis in veterinary medicine. Clin Microbiol Rev 14, 489512.

Hilborn, E. D., Yakrus, M. A., Covert, T. C., Harris, S. I., Donnelly, S. F., Schmitt, M. T., Toney, S., Bailey, S. A. \& Stelma, G. N., Jr (2008). Molecular comparison of Mycobacterium avium isolates from clinical and environmental sources. Appl Environ Microbiol 74, 4966-4968.

Hunter, P. R. \& Gaston, M. A. (1988). Numerical index of the discriminatory ability of typing systems: an application of Simpson's index of diversity. J Clin Microbiol 26, 2465-2466.

Kasai, H., Ezaki, T. \& Harayama, S. (2000). Differentiation of phylogenetically related slowly growing mycobacteria by their gyrB sequences. J Clin Microbiol 38, 301-308.

Komijn, R. E., de Haas, P. E., Schneider, M. M., Eger, T., Nieuwenhuijs, J. H., van den Hoek, R. J., Bakker, D., van Zijd Erveld, F. G. \& van Soolingen, D. (1999). Prevalence of Mycobacterium avium in slaughter pigs in The Netherlands and comparison of IS 1245 restriction fragment length polymorphism patterns of porcine and human isolates. J Clin Microbiol 37, 12541259.

Kumar, S., Tamura, K. \& Nei, M. (2004). MEGA3: integrated software for Molecular Evolutionary Genetics Analysis and sequence alignment. Brief Bioinform 5, 150-163.

Kyriakopoulos, A. M., Tassios, P. T., Matsiota-Bernard, P., Marinis, E., Tsaousidou, S. \& Legakis, N. J. (1997). Characterization to species level of Mycobacterium avium complex strains from human immunodeficiency virus-positive and -negative patients. J Clin Microbiol 35, 3001-3003.

Le Dantec, C., Duguet, J. P., Montiel, A., Dumoutier, N., Dubrou, S. \& Vincent, V. (2002). Occurrence of mycobacteria in water treatment lines and in water distribution systems. Appl Environ Microbiol 68, $5318-5325$.

Mazurek, G. H., Hartman, S., Zhang, Y., Brown, B. A., Hector, J. S., Murphy, D. \& Wallace, R. J., Jr (1993). Large DNA restriction fragment polymorphism in the Mycobacterium avium-M. intracellulare complex: a potential epidemiologic tool. J Clin Microbiol 31, 390-394.

Mijs, W., de Haas, P., Rossau, R., van der Laan, L., Rigouts, L., Portaels, F. \& van Soolingen, D. (2002). Molecular evidence to support a proposal to reserve the designation Mycobacterium avium subsp. avium for bird-type isolates and ' $M$. avium subsp. hominissuis' for the human/porcine type of M. avium. Int J Syst Evol Microbiol 52, 1505-1518.

Morita, Y., Maruyama, S., Kabeya, H., Nagai, A., Kozawa, K., Kato, M., Nakajima, T., Mikami, T., Katsube, Y. \& Kimura, H. (2004). Genetic diversity of the dnaJ gene in the Mycobacterium avium complex. J Med Microbiol 53, 813-817.

Murcia, M. I., Tortoli, E., Menendez, M. C., Palenque, E. \& Garcia, M. J. (2006). Mycobacterium colombiense sp. nov., a novel member of the Mycobacterium avium complex and description of MAC-X as a new ITS genetic variant. Int J Syst Evol Microbiol 56, 2049-2054.

Ning, Z., Cox, A. J. \& Mullikin, J. C. (2001). SSAHA: a fast search method for large DNA databases. Genome Res 11, 1725-1729.

Nishiuchi, Y., Maekura, R., Kitada, S., Tamaru, A., Taguri, T., Kira, Y., Hiraga, T., Hirotani, A., Yoshimura, K. \& other authors (2007). The recovery of Mycobacterium avium-intracellulare complex (MAC) from the residential bathrooms of patients with pulmonary MAC. Clin Infect Dis 45, 347-351.

Pestel-Caron, M., Graff, G., Berthelot, G., Pons, J. L. \& Lemeland, J. F. (1999). Molecular analysis of Mycobacterium avium isolates by using pulsed-field gel electrophoresis and PCR. J Clin Microbiol 37, 24502455.

Reed, C., von Reyn, C. F., Chamblee, S., Ellerbrock, T. V., Johnson, J. W., Marsh, B. J., Johnson, L. S., Trenschel, R. J. \& Horsburgh, C. R., Jr (2006). Environmental risk factors for infection with Mycobacterium avium complex. Am J Epidemiol 164, 32-40.

Santos, R., Oliveira, F., Fernandes, J., Gonçalves, S., Macieira, F. \& Cadete, M. (2005). Detection and identification of mycobacteria in the Lisbon water distribution system. Water Sci Technol 52, 177-180.

Thierry, D., Bauge, S., Poveda, J. D., Vincent, V. \& Guesdon, J. L. (1993). Rapid identification of Mycobacterium avium-intracellulare complex strains: clinical practice evaluation of DT6 and DT1 probes. $J$ Infect Dis 168, 1337-1338.

Thompson, J. D., Gibson, T. J., Plewniak, F., Jeanmougin, F. \& Higgins, D. G. (1997). The CLUSTAL_X Windows interface: flexible strategies for multiple sequence alignment aided by quality analysis tools. Nucleic Acids Res 25, 4876-4882.

Thorel, M. F., Krichevsky, M. \& Levy-Frebault, V. V. (1990). Numerical taxonomy of mycobactin-dependent mycobacteria, emended description of Mycobacterium avium, and description of Mycobacterium avium subsp. avium subsp. nov., Mycobacterium avium subsp. paratuberculosis subsp. nov., and Mycobacterium avium subsp. silvaticum subsp. nov. Int J Syst Bacteriol 40, 254-260.

Tortoli, E., Rindi, L., Garcia, M. J., Chiaradonna, P., Dei, R., Garzelli, C., Kroppenstedt, R. M., Lari, N., Mattei, R. \& other authors (2004). Proposal to elevate the genetic variant MAC-A, included in the Mycobacterium avium complex, to species rank as Mycobacterium chimaera sp. nov. Int J Syst Evol Microbiol 54, 1277-1285.

Turenne, C. Y., Semret, M., Cousins, D. V., Collins, D. M. \& Behr, M. A. (2006). Sequencing of hsp65 distinguishes among subsets of the Mycobacterium avium complex. J Clin Microbiol 44, 433-440.

Turenne, C. Y., Wallace, R. \& Behr, M. A. (2007). Mycobacterium avium in the postgenomic era. Clin Microbiol Rev 20, 205-229.

Turenne, C. Y., Collins, M., Alexander, D. C. \& Behr, M. A. (2008). Mycobacterium avium subsp. paratuberculosis and M. avium subsp. avium are independently evolved pathogenic clones of a much broader group of M. avium organisms. J Bacteriol 190, 2479-2487.

Vuorenmaa, K., Ben Salah, I., Barlogis, V., Chambost, H. \& Drancourt, M. (2009). Mycobacterium colombiense and pseudotuberculous lymphadenopathy. Emerg Infect Dis 15, 619-620.

Edited by: G. R. Stewart 